\title{
Using paraxial approximation to describe the optical setup of a typical EARLINET lidar system
}

\author{
Panagiotis Kokkalis \\ Institute of Astronomy, Astrophysics, Space Applications and Remote Sensing, National Observatory of Athens, \\ 15236, Greece \\ Correspondence to: Panagiotis Kokkalis (panko@noa.gr)
}

Received: 9 July 2016 - Discussion started: 2 August 2016

Revised: 3 June 2017 - Accepted: 10 July 2017 - Published: 25 August 2017

\begin{abstract}
The mathematical formulation for the optical setup of a typical EARLINET lidar system is given here. The equations describing a lidar system from the emitted laser beam to the projection of the telescope aperture on the final receiving unit (i.e., photomultiplier or photodiode) are presented, based on paraxial approximation and geometric optics approach. The receiving optical setup includes a telescope, a collimating lens, an interference filter and the ensemble objective eyepiece. The set of the derived equations interconnects major parameters of the optical components (e.g., focal lengths, diameters, angles of incidence), revealing their association with the distance of full overlap of the system. These equations may used complementarily with an optical design software, for the preliminary design of a system or can be used as a quick check up tool of an existing lidar system. The evaluation of the formulation on a real system is performed with ray-tracing simulations, revealing an overall good performance with relative differences of the order of $5 \%$ mainly attributed to the limitations of the thin lens approximation.
\end{abstract}

\section{Introduction}

Lidars are efficient tools for retrieving the aerosol optical and microphysical properties in the planetary boundary layer (PBL) and free troposphere. More precisely, the lidar techniques that are widely used for aerosol research are capable of providing range-resolved information for (a) the aerosol backscatter coefficient ( $\beta_{\alpha \mathrm{er}}$ ) using the backscatter lidar technique (e.g., Fernald et al., 1972; Klett, 1981), (b) the aerosol extinction coefficient $\left(\alpha_{\alpha \mathrm{er}}\right)$ using the Raman lidar technique (Ansmann et al., 1990, 1992) and (c) the volume and particle linear depolarization ratios using the depolarization lidar technique (e.g., Sassen, 2005; Freudenthaler et al., 2009). Many studies have demonstrated that the provision of the aforementioned aerosol optical properties for multiple wavelengths facilitates the retrieval of aerosol microphysical properties through inversion techniques (Müller et al., 1999; Veselovskii et al., 2002, 2010). The accuracy of the optical properties used as inputs for the inversions is critical. Uncertainties involved for the retrieval of the aerosol optical properties with lidar techniques are linked both to systematic and statistical sources of error. Statistical errors are due to noise associated to the photon nature of the signal itself and to noise added in the detection process (Ansmann et al., 1992; Bösenberg, 1997; Iarlori et al., 2015). On the other hand, systematic errors may arise both from assumptions or uncertain values that enter the lidar data analysis and from the system setup and geometry. The first category may include uncertainties introduced by the estimation of temperature and pressure profiles along with the wavelength dependence parameter required in the Raman technique (Ansmann et al., 1990; Whiteman, 1999) and the assumption of lidar ratio, reference height and backscattering ratio, required in the backscatter technique. The second broad category of systematic errors may include uncertainties introduced, for example, by interferences caused by the laser source in the analog receiving channels, the range-dependent overlap factor, the calibration of the system, etc. Over the last decades, a lot of work towards estimating and minimizing the errors in aerosol lidar retrievals has been done in the framework of the European Aerosol Research Lidar Network (EARLINET; Pappalardo et al., 2014). For example, and in order to optimize the opti- 
cal performance and control the quality of aerosol measurements, a number of quality assurance (QA) tests have been adopted and applied in EARLINET lidar systems (Freudenthaler, 2008). Moreover, increased effort has been made by the European lidar community to develop and apply accurate depolarization calibration techniques (Freudenthaler, 2016) and quantify and correct the influence of systematic error introduced by imperfections of lidar optical elements on the depolarization related retrievals (Mattis et al., 2009; BravoAranda et al., 2016; Belegante et al., 2016). These studies are based on the description of the state of polarization of light and lidar optical elements by means of the MüllerStokes formulation. The present study tackles basic lidar design trade-offs, and more advanced topics such as depolarization measurements are out of its scope.

The lidar equation in its simplest form includes the overlap function $(O(z))$ and the overall optical efficiency of the system. The overlap function is range-dependent and thus related to the lidar system geometry, since it describes the fraction of the light scattered within the receiver field of view, taking values from 0 to 1 (Wandinger, 2005). More precisely, at the height range where the overlap function reaches the value of 1 , each point of the telescope aperture collects the scattered light entirely and with the same efficiency (Fig. 1a). This height range is determined by the intersection point between the outer edge of the laser beam divergence (LBD) and the lateral surface of receiver field of view (RFOV) cone, with the apex on the point of the telescope aperture farthest away from the laser (Fig. 1a). This range is known as the distance of full overlap (DFO) and is usually found from 500 to $1500 \mathrm{~m}$ for EARLINET aerosol lidar systems. The overlap function depends on the range up to the DFO, adding a significant drawback to the retrieval of aerosol optical properties from lidar systems, since it becomes difficult to obtain useful and accurate information regarding the aerosol present below that height range. More precisely, Wandinger and Ansmann (2002) demonstrated that when not applying overlap correction in lidar signals, the retrieved aerosol extinction coefficient may take even non-physical negative values for heights up to the DFO. However, they proposed that the effect of the incomplete overlap can be corrected, and trustworthy retrievals may finally be obtained in the case of a system operating both elastic and Raman channels under the assumption that they are affected by the same overlap function.

Thus, in order to optimize the performance of a lidar at lower altitudes and effectively retrieve optical properties of the aerosol entrapped below the PBL height, it is of great importance that the receiving telescope is able to detecting the emitted laser pulse, already at short ranges from the lidar system. Therefore, a low full overlap height is needed. The wide-angle RFOV is not an optimal solution for minimizing the DFO, since in that case (a) the signal will be contaminated with more sky background light, and (b) multiple scattering effects have to be taken into account, especially for case studies of optically thick targets (i.e., water and ice clouds; e.g., Eloranta, 1998; Wandinger, 1998). Nevertheless, there are systems employing two telescopes, one for the short ranges and the other for the far ones, with the short-range one having a wider RFOV angle (e.g., Engelmann et al., 2016). However, such setups are more complicated and their signals demand special treatment during the retrieval processing schemes.

Case studies, but also long-term lidar observations performed during the last decade at various EARLINET stations, revealed that the DFO have to be much lower than $600 \mathrm{~m}$ in order to detect the boundary layer at European latitudes, especially during wintertime (e.g., Matthias and Bösenberg, 2002; Matthias et al., 2004; Amiridis et al., 2007; Baars et al., 2008). Measurements of the aerosols within the $\mathrm{PBL}$ are in particular required during daytime, when the convection is stronger. However, daytime lidar operation suffers from the increased sky radiance contaminating the lidar signal, which needs suppression. In order to suppress the bright daytime sky radiance and enhance the signal to noise ratio (SNR) of the lidar signal, small bandwidth (BW) interference filters (IFFs) are widely used in EARLINET. Such IFFs have recently become commercially available with small BW narrower than $0.2 \mathrm{~nm}$ (FWHM) at the visible spectrum and high transmission values (greater than 90\%) at peak (Alluxa, CA, http://www.alluxa.com). Their high transmission and narrow BW characteristics have been recently used for rotational Raman measurements at visible (Veselovskii et al., 2015) and infrared spectrum (Haarig et al., 2016). A significant drawback of these filters is that their narrow bandwidth can cause low acceptance angle $\left(A_{\mathrm{IFF}}^{\max }\right)$, which in turn limits the possible DFO. Alternative methods for efficiently suppressing the background are based on the shaping of the receivers field of view diaphragm (FOVD) along with their geometry and their relative position on the optical axis, as has been proposed by Abramochkin and Tikhomirov (1999) and Freudenthaler (2003).

There are several studies in the literature related to the determination of the overlap function of lidar systems analytically (e.g., Halldórsson and Langerholc, 1978; Jenness et al., 1997; Chourdakis et al., 2002; Stelmaszczyk et al., 2005; Comeron et al., 2011) or experimentally (e.g., Sasano et al., 1979; Tomine et al., 1989; Dho et al., 1997; GuerreroRascado et al., 2010; Wandinger and Ansmann, 2002). For the theoretical approaches, a good understanding of the actual light distribution in the laser beam cross section, and the characteristics of the receiving unit are needed to obtain an overlap profile with sufficient accuracy. Stelmaszczyk et al. (2005) also proposed an analytical formula to decrease the DFO based only on the laser telescope geometry and specifically the introduction of a small inclination between the transmitter and receiver central axis. However, all the aforementioned theoretical studies provide information regarding the overlap function based explicitly on the laser telescope setup. 
(a)
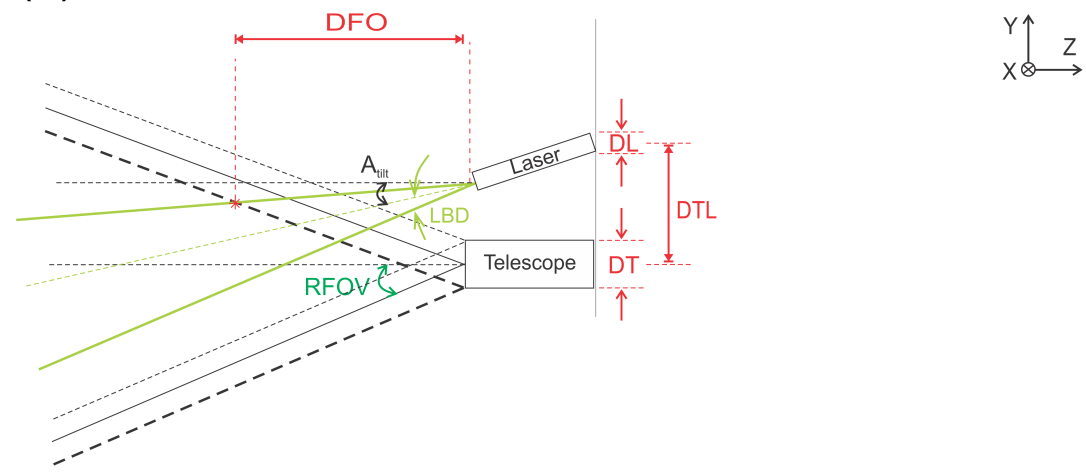

(b)

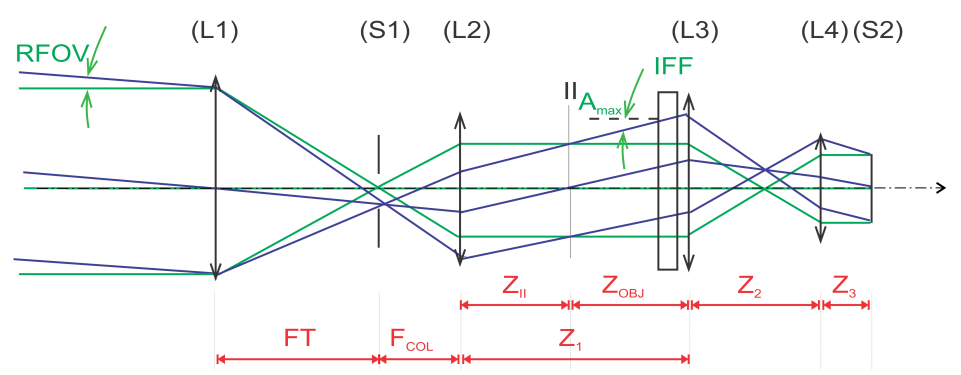

Figure 1. (a) The laser telescope geometry of a biaxial lidar system with a laser tilt $A_{\text {tilt }}$ and distance of full overlap DFO. RFOV and LBD are the receiver's field of view and laser beam divergence respectively (half angles). Due to the small angle approximation, the errors are negligible when defining the laser beam diameter (DL), as if the laser was not tilted, and the distance between the laser telescope central axes (DTL) at the back of the laser and telescope. (b) The optical setup of a lidar receiving unit with a telescope (L1), field of view diaphragm LBD (S1), collimating lens (L2), interference filter and objective lens (L3) and an eyepiece lens (L4). Rays collected from on-axis (green lines) and off-axis points (blue lines) with the maximum incident angle at the telescope (RFOV), which is limited by the FOVD, reaches the IFF surface with a free aperture diameter of $D_{\mathrm{obj}}$, located at distance $Z_{1}$ from L2 under an incident angle $A_{\text {max }}$. S2 is the surface of the PMT with diameter $D_{\mathrm{PMT}}$.

This study focuses on the extension of the paraxial approximation down to the detector, revealing all the possible constraints of a lidar setup, since DFO depends on the overall optical path of the detected backscattered radiation. The distance of full overlap, as presented in this work, depends on the entire geometry considering all the parts of the lidar as one optical system. The analysis in this study highlights the need to take into consideration the acceptance angle of the interference filter when designing an optimized lidar system and the possible limitations that this imposes. The corresponding geometrical formulation is presented in Sect. 2, describing the basic characteristics (focal lengths, distances and diameters) of all the optical components, compromised with the EARLINET QA standards. The derived formulation also includes the characteristics of an eyepiece lens, which has to be used in a lidar setup in order to form an image of the entrance pupil on the surface of the photodetector so as to spread the collected light uniformly over this image. The results of ray-tracing simulations with respect to lidar design and alignment according to geometrical formulation are presented in Sect. 3.
The entire set of equations has been integrated in a Microsoft Excel worksheet through Visual Basic for Applications (VBA) code and distributed with supplementary documentation to the members of EARLINET network (http: //www.meteo.physik.uni-muenchen.de/ stlidar/earlinet_ asos/raytracing/Basic_design/basic_lidar_design.html), but can also be widely available upon request from the author (panko@noa.gr). The aforementioned worksheet cannot substitute advanced optical design software. However, it may be complementary to the preliminary design of a system, can be used as a quick checkup tool for an existing lidar system or even used as a learning tool to familiarize a system with an optical lidar setup.

\section{Lidar optical setup and limitations}

Lidar systems are using large telescopes to collect the weak light, backscattered from the atmosphere. This portion of light has to be further transmitted and projected to small detectors without any range-dependent losses. For example, with a telescope diameter of $300 \mathrm{~mm}$ and a detector diame- 
ter of $5 \mathrm{~mm}$, an overall magnification of the optical system of 0.0166 is necessary. On the other hand, the angular magnification is increased by 60 , which means that $1.25 \mathrm{mrad}$ field of view of the receiver setup (i.e., determined by telescope with focal length $600 \mathrm{~mm}$ and a field stop with diameter $1.5 \mathrm{~mm}$ ), is magnified to about $75 \mathrm{mrad}\left(\sim 4.3^{\circ}\right)$.

For single but more particularly for multiwavelength systems, the wavelength separation unit is not capable of accepting such a divergent received light beam, since (a) it would soon be too wide for 1 or 2 inch optical elements, and (b) the transmission of interference filters is very sensitive to the incidence angle. Therefore, the magnification of the receiver optics is split in two parts, i.e., the telescope with a collimation lens and another objective with an eyepiece, with a low divergent light path (parallel received light beam) in between (Fig. 1b). The inclination of the parallel beam is determined by the laser beam divergence, the laser telescope axis distance, the tilt of the laser beam with respect to the telescope axis, determining the field of incidence angles into the telescope, and the magnification of the telescope together with the collimating lens.

The limitations for this inclination of the received rays are the following: the field of view of the telescope should be as small as possible in order to reduce the background light collected from the sky; the laser beam diameter and its divergence must both be small enough to fit through the 1 inch optics for all necessary beam splitters; the inclination must be less than the maximum acceptance angles of the interference filters.

\subsection{Lidar optical layout}

The setup of a biaxial lidar system is schematically given in Fig. 1. The laser telescope geometry is demonstrated in the upper part (Fig. 1a) while the optical setup of the lidar receiving unit behind the telescope, is presented in the lower part (Fig. 1b). The abbreviations used in this study in order to describe the lidar parameters are summarized in the Appendix as a list of abbreviations.

The modeling of a transmitted laser beam in the atmosphere has been approximated by a truncated cone of an ideally circularly shaped beam with initial diameter DL and divergence LBD (half angle). The DL and LBD values provided by the manufacturers usually correspond to the $86.5 \%$ $(2 \sigma)$ of the Gaussian beam energy. In lidar optical systems, the highest possible of the laser energy is needed and, to account for a Gaussian laser beam containing the $98.9 \%(3 \sigma)$ of the beam energy, both DL and LBD have to be reduced by a factor of 0.5 . The laser beam interacts with the atmospheric constituents (aerosols and molecules) and the backscattered light is collected by a telescope with a focal length FT and clear aperture DT. The distance between the transmitter and receiver central axes is DTL (Fig. 1a). The diaphragm is usually a circular iris, with diameter $D_{\mathrm{FS}}$ centered on the optical axis, and mounted on telescope's focal plane. The fo- cal length of the telescope and the diaphragm FOVD determine the RFOV (half angle) of the receiver setup, according to $\mathrm{RFOV}=\frac{D_{\mathrm{FS}}}{2 \times \mathrm{FT}}$.

For simplicity, all the optical components of the system (telescope and lenses) are presented as thin lenses in Fig. 1b. In addition to the paraxial approximation assumption, namely that rays are not too distant from the system axis and their angles with respect to that axis are small, it also implies that aberration effects are not considered.

Regardless of the origin point of the incoming rays, any rays incident at the telescope with angles higher than RFOV will not pass through the diaphragm (FOVD; S1). Such a scenario is demonstrated in Fig. 1b where two extreme lines are shown: a green line representing rays parallel to the axis passes through the center of the field stop and normally impinges on the interference filter and a blue line which represents extreme rays grazes the edge of the field stop at the maximum angle with respect to the normal to the interference filter (Fig. 1b). The entire range of angles of rays passing through any point of the telescope aperture and any point of the field stop must be limited by the collimating lens (L2; collimator) with diameter $D_{\mathrm{col}}$ and focal length $F_{\mathrm{col}}$, mounted at distance $F_{\text {col }}$ behind the field stop. The collimation of the rays is mandatory due to the limited acceptance angles $A_{\mathrm{IFF}}^{\max }$. of the IFFs (see Appendix). The collimating lens (L2) produces an intermediate image (II) of the entrance pupil, at a distance $Z_{\mathrm{II}}$ behind that lens. More precisely, the intermediate image is formed at the so called eye-relief plane, where rays with different inclinations passing through the same points of the aperture cross each other. In the optical setup presented here, this plane appears twice; firstly behind the collimator and secondly behind the eyepiece. Regarding the first position, in case an optical detection device (e.g., PMT) is mounted there, it will collect all the power carried by rays reaching the aperture within the field of view, provided that its diameter is larger (or at least equal) than the image of the aperture formed by the collimating lens on that plane. This requires detection devices of large aperture (e.g., 1-2 inches) and consequently of high cost. However, by using an additional optical system assembled from an objective (L3) and an eyepiece lens (L4), this image will be formed again behind the eyepiece to a lower diameter. An objective lens (L3) with focal length $F_{\text {obj }}$ and diameter $D_{\text {obj }}$ is located just behind the IFF and at a distance $Z_{1}$ behind the collimator. An eyepiece lens (L4) with focal length $F_{\text {eye }}$ and diameter $D_{\text {eye }}$ is placed at a distance of $Z_{2}=F_{\text {obj }}+F_{\text {eye }}$ behind the objective lens. The focal length of the objective lens must not be shorter than three times its diameter, since for low $F_{\text {obj }}$ values of a simple, planoconvex or biconvex lens, the final image of the telescope aperture on the photomultiplier (PMT) may be affected by aberration effects. The PMT (S2) with diameter $D_{\text {PMT }}$ is located at distance $Z_{3}$ behind the eyepiece lens and on its surface the image of the clear aperture of the telescope is projected. The aforementioned components have to fulfil specific conditions regarding their diameter and focal length 
and should be accurately mounted on the optical path of the collected backscattered light in order to achieve an optimum imaging (e.g., avoiding vignetting effects, keeping the condition that $A_{\max } \leq A_{\mathrm{IFF}}^{\max }$ ) of the telescope's aperture onto the detector's effective surface.

In principle, the system presented in Fig. $1 \mathrm{~b}$ spreads uniformly over the image of the entrance aperture formed on the photodetector surface the light coming from the illuminated parts of the atmosphere within the system field of view. The effective diameter of the photomultiplier is maximum $8 \mathrm{~mm}$ for Hamamatsu PMTs R7400 series (Hamamatsu Photonics, 2006). However, the useful diameter of the PMT is about $5 \mathrm{~mm}$, including mounting and adjustment tolerances.

For ranges above the DFO, the laser beam stays entirely inside the telescope's full field of view. For those ranges, any ray coming from a point in the illuminated volume and reaching the telescope aperture will pass through the field stop diaphragm.

\subsection{Description through paraxial approximation}

The parameter of RFOV is chosen as the coupling link between the laser telescope part and the detection optics, which are located after the telescope focus. This choice can be explained, since on one side the given telescope and laser geometrical characteristics determine the DFO, and on the other side towards the PMT, all rays entering the field stop have to be collected by the PMT.

As also demonstrated from Stelmaszczyk et al. (2005) from Fig. 1a we have the following:

$\mathrm{DFO}=\frac{2 \times \mathrm{DTL}+\mathrm{DT}+\mathrm{DL}}{2 \times\left(\mathrm{RFOV}-\mathrm{LBD}+A_{\mathrm{tilt}}\right)}$.

In the case that a laser beam expander with an expansion factor of EX is used in the emission part of a biaxial lidar configuration, the initial laser diameter increases and the corresponding laser beam divergence decreases, by a factor of EX. Thus, the effective laser parameters (DL and LBD) after the expansion will become respectively, DL $\times \mathrm{EX}$ and $\mathrm{LBD} \times \mathrm{EX}^{-1}$, in all formulas. The above are approximations and hold true for ideal optical components, since in general, commercial laser beam expanders demonstrate different efficiencies regarding the expansion of the laser beam diameter and the reduction of the laser beam divergence.

The dominator of Eq. (1) must be positive (i.e., RFOV $\mathrm{LBD}+A_{\text {tilt }} \geq 0$ ) in order to have DFO $\geq 0$. This, together with the condition that $A_{\text {tilt }}+\mathrm{LBD} \leq \mathrm{RFOV}$ leads to the basic principle for lidar applications that the receiver's field of view cannot be smaller than the laser beam divergence (i.e., $\mathrm{RFOV} \geq \mathrm{LBD}$ ). In the case that the condition $A_{\text {tilt }}+\mathrm{LBD} \leq \mathrm{RFOV}$ is not fulfilled, even if the full overlap is reached in some range, the laser beam will eventually exit the full field of view zone in the far range. With paraxial optics and small angle approximation, we can extract from Fig. 1b the following relation:

$\mathrm{RFOV}=\frac{D_{\mathrm{FS}}}{2 \times \mathrm{FT}}=\frac{F_{\mathrm{col}}}{\mathrm{FT}} \times A_{\max }$,

where $A_{\max }$ is the maximum incidence angle on the interference filter of rays passing through the field stop. This angle should be less than or equal to the maximum acceptance angle of the interference filter:

$A_{\max } \leq A_{\mathrm{IFF}}^{\max }$.

The main goal when designing a lidar system for aerosol research in the lower to middle troposphere is to make the DFO as short as possible while keeping (a) the incidence angle of the rays on the interference filter surface less than its acceptance angle (i.e., $A_{\max } \leq A_{\mathrm{IFF}}^{\max }$ ) and (b) the diameters of the lenses within reasonable values. For the use of a small bandwidth IFF with small $A_{\mathrm{IFF}}^{\max }$ it is necessary to keep the RFOV small or to increase the ratio $\frac{F_{\mathrm{col}}}{\mathrm{FT}}$. In biaxial lidar systems the RFOV is determined by the parameters of the receiver setup (FT and $D_{\mathrm{FS}}$ ), and the higher the RFOV, the lower the DFO ranges that may be achieved (Eq. 1). In addition, by increasing the $D_{\mathrm{FS}}$ the RFOV increases (Eq. 2) and the DFO decreases but the SNR becomes lower, especially during daytime conditions when the detected lidar signal is contaminated with more light from the sky background. Moreover, high RFOV values tend to increase the diameters of the lenses in the receiving optical setup.

With $A_{\mathrm{IFF}}=A_{\max }$ and for any $A_{\text {tilt }}$ from Fig. $1 \mathrm{~b}$ and Eqs. (1) and (2) we get

$\mathrm{DFO}=\frac{2 \times \mathrm{DTL}+\mathrm{DT}+\mathrm{DL}}{2 \times\left(\frac{F_{\mathrm{col}}}{\mathrm{FT}} \times A_{\max }-\mathrm{LBD}+A_{\mathrm{tilt}}\right)}$.

The ratio $\frac{F_{\mathrm{col}}}{\mathrm{FT}}$ is limited by the diameters of $D_{\mathrm{col}}$ and DT (compare Fig. 1b) by

$D_{\mathrm{col}} \geq \mathrm{DT} \times \frac{F_{\mathrm{col}}}{\mathrm{FT}}+2 \times \mathrm{RFOV} \times\left(\mathrm{FT}+F_{\mathrm{col}}\right)$.

Consequently,

$\frac{\left(\frac{D_{\mathrm{col}}}{2}-\mathrm{RFOV} \times \mathrm{FT}\right)}{F_{\mathrm{col}}} \geq \frac{\left(\frac{\mathrm{DT}}{2}+\mathrm{RFOV} \times \mathrm{FT}\right)}{\mathrm{FT}}$.

Additional constraints are the limited diameters of the lenses, filters and beam splitters in combination with the diameter and the focal lengths of the telescope and the collimator (i.e., FT and $F_{\mathrm{col}}$ ), as well as the distance $Z_{1}$ which needs to be as high as possible to mount all the optical elements, especially for the case of multiwavelength backscatter Raman 
lidar systems. Note, that the diameter $D_{\text {col }}$ of the optical parts is limited by their rising cost with diameter and decreasing availability. The extreme rays in Fig. 1b must pass through all the optics, which results in Eq. (6), expressed here for the minimum and maximum focal length of the telescope with given RFOV.

$$
\begin{aligned}
& \mathrm{FT}_{\max / \min }=0.5 \times\left(\frac{D_{\mathrm{col}}}{2 \times \mathrm{RFOV}}-F_{\mathrm{col}}\right) \\
& \pm \sqrt{0.25 \times\left(F_{\mathrm{col}}-\frac{D_{\mathrm{col}}}{2 \times \mathrm{RFOV}}\right)^{2}-\frac{F_{\mathrm{col}} \times \mathrm{DT}}{2 \times \mathrm{RFOV}}}
\end{aligned}
$$

All these parameters must be balanced for optimum lidar performance and for a specific scientific objective. The following system of equations (Eq. 8) is derived with paraxial approximation (Fig. 1b). The first one is given by definition (Fig. 1b), the second one gives the plane where the image of the entrance pupil by the collimating lens is formed, and the third one gives the minimum diameter that the objective lens must have in order to let all the rays within the field of view pass:

$$
\begin{aligned}
& Z_{1}=Z_{\mathrm{obj}}+Z_{\mathrm{II}} \\
& Z_{\mathrm{II}}=\frac{\mathrm{RFOV}}{A_{\max }} \times\left(F_{\mathrm{col}}+\mathrm{FT}\right) \\
& \frac{D_{\mathrm{obj}}}{2}=\frac{\mathrm{DT} \times \frac{F_{\mathrm{col}}}{\mathrm{FT}}}{2}+A_{\max } \times Z_{\mathrm{obj}} \\
& \stackrel{\text { yields }}{\longrightarrow} Z_{1}=\frac{1}{2 \times A_{\max }} \\
& \times\left[D_{\mathrm{obj}}-\mathrm{DT} \times \frac{F_{\mathrm{col}}}{\mathrm{FT}}+2 \times \mathrm{RFOV} \times\left(F_{\mathrm{col}}+\mathrm{FT}\right)\right] .
\end{aligned}
$$

Furthermore, in the $Z_{\text {II }}$ expression that appears in the block of Eq. (8), the term $\frac{R F O V}{A_{\max }}$ has been substituted for $\frac{F_{\mathrm{col}}}{\mathrm{FT}}$. Following Figs. $1 \mathrm{~b}$ and $2 \mathrm{a}$, the diameter of the intermediate image $\left(D_{\text {II }}\right)$ formed on the eye-relief plane between the collimator and the objective lens, and the diameter of the objective lens $\left(D_{\text {obj }}\right)$ just behind the IFF are equal to

$$
\begin{aligned}
D_{\mathrm{II}} & =\frac{\mathrm{DT}}{\mathrm{FT}} \times F_{\mathrm{col}} \\
D_{\mathrm{obj}} & =D_{\mathrm{II}}+2 \times A_{\max } \times Z_{\mathrm{obj}} .
\end{aligned}
$$

The rays collected by the IFF and the objective lens (L3 in Fig. 1b) are guided through the eyepiece lens (L4 in Fig. 1b), creating the final image of the entrance pupil at a distance $Z_{3}$ behind the last surface of the eyepiece lens. More precisely, the intermediate image of the telescope aperture (with diameter $\left.D_{\mathrm{II}}\right)$ is formed initially at distance $Z_{\mathrm{obj}}$ before the objective lens (L3; Figs. 1b and 2). In the setup presented in Fig. 2, the intermediate image is now the object with diameter $D_{\text {II }}$ that has to be projected on the surface of the photodetector (S2) through the system of two lenses. The first lens of this system is an objective lens (L3) with focal length $F_{\mathrm{obj}}$, and the second one is an eyepiece lens (L4) with focal

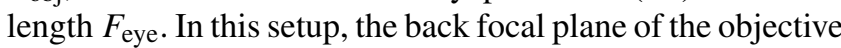
and the front focal plane of the eyepiece coincide; i.e., the separation between the objective and the eyepiece is equal to $Z_{2}=F_{\text {obj }}+F_{\text {eye }}$ The total magnification of that lens system is $M_{3,4}=\frac{F_{\text {eye }}}{F_{\text {obj }}}$. Therefore, the final image of the object $\left(D_{\mathrm{II}}\right)$ projected on (S2) would have the following diameter (Fig. 2a):

$D_{\mathrm{PMT}} \geq M_{3,4} \times D_{\mathrm{II}} \rightarrow F_{\text {eye }} \leq \frac{D_{\mathrm{PMT}}}{D_{\mathrm{II}}} \times F_{\text {obj }}$.

From the similarity of the triangles shown in Fig. 2b we find that $R_{3}=\frac{R_{1}}{F_{\mathrm{obj}}} \times F_{\text {eye }}$, where $R_{1}$ is the diameter of an object produced by of off axis rays on the intermediate plane (Fig. 2b). The free aperture of the lens (L4) in order to collect both off and on-axis points of $D_{\text {II }}$ has to be $D_{\text {eye }}$ (Fig. 2b):

$$
D_{\text {eye }}=2 \times\left[A_{\max } \times F_{\mathrm{obj}}+\frac{\frac{D_{\mathrm{II}}}{2}-A_{\max } \times\left(Z_{\mathrm{obj}}-F_{\mathrm{obj}}\right)}{F_{\mathrm{obj}}} \times F_{\text {eye }}\right] .
$$

Considering as object the image of the aperture by L2, which is at distance $Z_{\mathrm{obj}}$ of L3, the afocal system of the lenses L3 and L4 will project it, at a distance $Z_{3}$ behind L4. This distance is

$Z_{3}=F_{\text {eye }} \times\left[1-\frac{F_{\text {eye }} \times\left(Z_{\text {obj }}-F_{\text {obj }}\right)}{F_{\text {obj }}^{2}}\right]$.

PMTs and APDs suffer from a non-uniform spatial response of their effective surface, which may cause artifacts to lidar signals during its transduction into electrical signal. Simeonov et al. (1999) revealed that the normalized spatial uniformity on the active area of the detector varies from 0.2 up to almost three times the average value, defined for the central part of the detector. In order to avoid lidar signal deviations due to the spatial inhomogeneity PMT sensitivity, the detector must be placed at an image of the telescopes aperture. At this place (distance $Z_{3}$ behind the eyepiece lens; L4), the system is forming the image of the telescope aperture, therefore spreading the light from illuminated points in the atmosphere uniformly over the photodetector surface (S2). In addition, an advantage of using an eyepiece lens is that the detection surface is rather insensitive to several axial/radial misalignments (e.g., $\pm 4 \mathrm{~mm} / \pm 2 \mathrm{~mm}$ ) of the lens L4 and the PMT (Freudenthaler et al., 2004). However, due to difficulties in measuring the exact location of the PMT cathode with respect to the PMT housing, the alignment of the detection surface behind the L4 seems to be crucial, and real ray tracing is necessary to determine the needed precision for the positioning of the photodetector surface. 

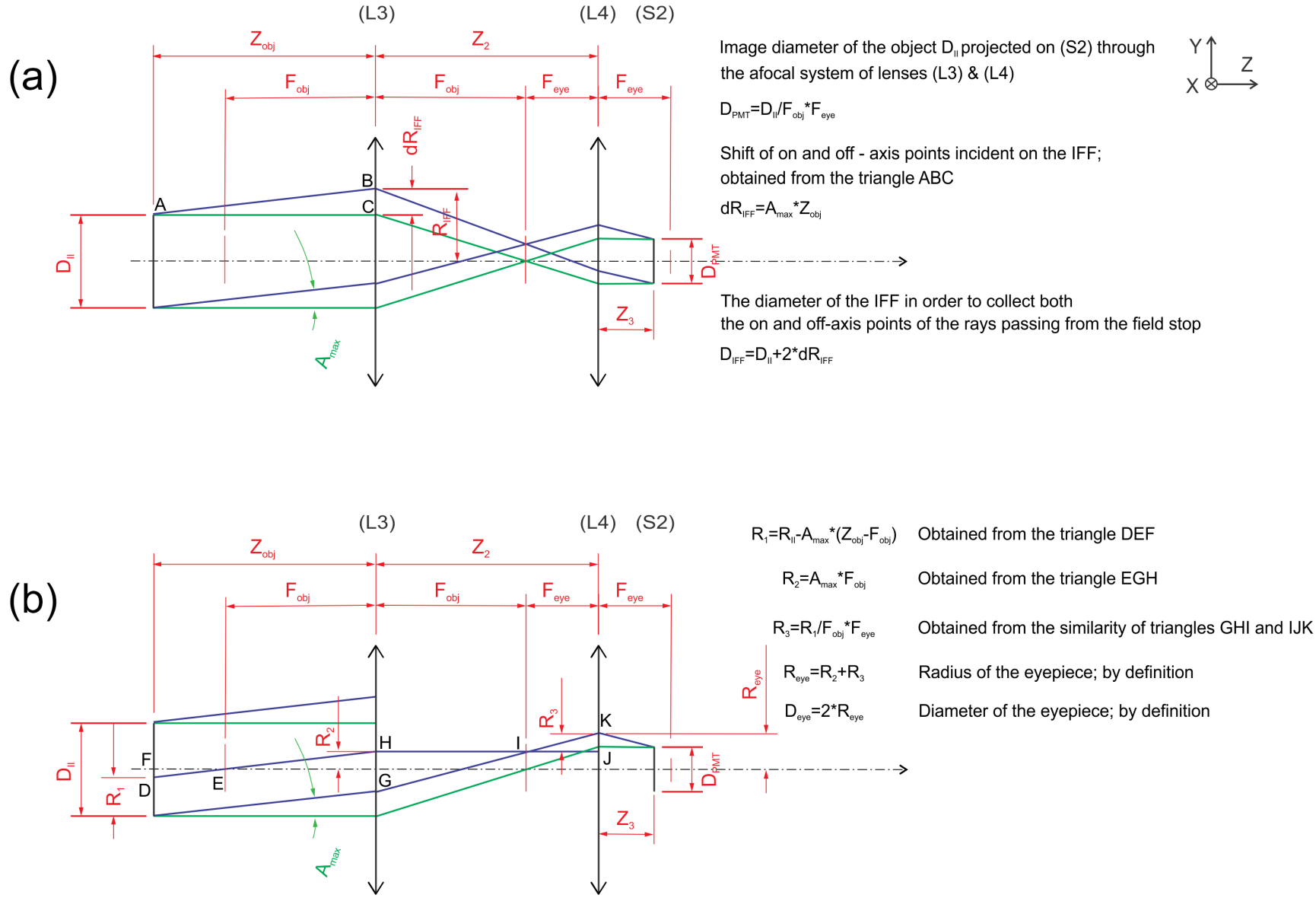

Figure 2. Panels (a) and (b) are essentially the same. However, to help the understanding of geometric calculations, (a) demonstrates the optical path of the on-axis (green line) and off-axis (blue line) points, the first intermediate image (II) of the telescope aperture with diameter $D_{\text {II }}$ formed at distance $Z_{\mathrm{obj}}$ before the objective lens (L3) and (b) the optical path of the on-axis (green line) and off-axis (blue line) points, of an object (with diameter $R_{1}$ ) produced by off-axis points on the intermediate plane. Through the objective lens (L3) with focal length $F_{\text {obj }}$ and an eyepiece lens (L4) with focal length $F_{\text {eye }}$ the intermediate image is formed again on the surface of the photodetector (S2), at a distance $Z_{3}$ behind the eyepiece lens. For reasons of simplicity the IFF is not included in this figure. The focal planes of each lens are denoted with vertical red lines on the principal axis.

For boundary layer measurements a low DFO height is required (see Fig. 1a), thus leading to higher values of $A_{\max }$ (Eq. 4), larger IFF bandwidth, lower sky background suppression and finally lower SNR of the system.

Tilting the laser by an angle $A_{\text {tilt }}$ with respect to the telescope axis (Fig. 1a), with the constraint that $A_{\text {tilt }} \leq$ RFOV LBD, allows a decrease of the RFOV with constant DFO or a decrease of the DFO with constant RFOV (Stelmaszczyk et al., 2005). The optimum $A_{\text {tilt }}$ is either the one that minimizes the RFOV or the DFO respectively, and for both aforementioned cases becomes equal to

$A_{\mathrm{tilt}}^{\mathrm{opt}}=\mathrm{RFOV}-\mathrm{LBD}$.

More clearly, for the former scenario
$A_{\mathrm{tilt}}^{\mathrm{opt}}=\frac{2 \times \mathrm{DTL}+\mathrm{DT}+\mathrm{DL}}{4 \times \mathrm{DFO}}$,

and with $A_{\text {tilt }}^{\text {opt }}$ according to Eq. (2)

$A_{\max }=\frac{\mathrm{FT}}{F_{\mathrm{col}}} \times\left[\frac{2 \times \mathrm{DTL}+\mathrm{DT}+\mathrm{DL}}{4 \times \mathrm{DFO}}+\mathrm{LBD}\right]$

The IFF allows for acceptable transmission of the backscattered rays with incident angles lower than $A_{\mathrm{IFF}}^{\max }$ (see Appendix). The smaller the filter bandwidth, the smaller the $A_{\mathrm{IFF}}^{\max }$ (a filter with bandwidth $\mathrm{BW}=0.5 \mathrm{~nm}$, leading to $\left.A_{\mathrm{IFF}}^{\max }=2.9^{\circ}\right)$. The extreme incident angles in the telescope (RFOV) and at the IFF $\left(A_{\max }\right)$ increase with decreasing DFO according to Eqs. (4) and (16). 


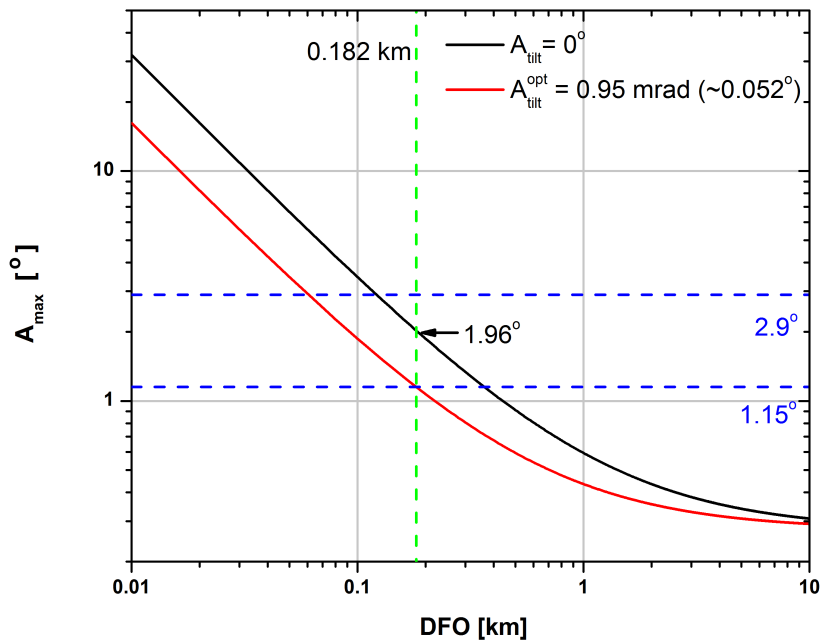

Figure 3. The variability of the maximum angle of incident rays on the IFF $\left(A_{\max }\right)$ for different DFO values, without laser tilt $\left(A_{\mathrm{tilt}}=\right.$ $0^{0}$; black line $)$ and with optimum laser tilt $\left(A_{\text {tilt }}^{\text {opt. }}=095 \mathrm{mrad}\right.$; red line). The blue horizontal dashed lines correspond to the maximum acceptance angles $\left(2.9\right.$ and $\left.1.15^{\circ}\right)$ of two IFFs with bandwidths of 0.5 and $0.15 \mathrm{~nm}$ respectively (see Appendix).

In Fig. 3 the variation of the maximum angle of incident rays on the IFF $\left(A_{\max }\right)$ for different DFO values is presented, regarding zero degrees and optimum laser tilt $\left(A_{\text {tilt }}^{\text {opt }}\right)$, according to Eqs. (4) and (16). The values used for the calculations (e.g., FT $F_{\text {col }}$ DTLDTDL) are provided in Sect. 3. The maximum angle of incident rays $\left(A_{\max }\right)$ on the IFF is decreased by about $40 \%$ (from 1.96 to $1.15^{\circ}$ ) with an optimum laser tilt for the same DFO $(182.11 \mathrm{~m})$. The two blue lines indicate the $A_{\mathrm{IFF}}^{\max }$ angles for two IFFs with BW 0.5 and $0.15 \mathrm{~nm}$ respectively (see Appendix).

\section{Evaluation of paraxial approximation with ray-tracing simulations}

For evaluating the formulation presented in this study, raytracing simulations with ZEMAX software (www.zemax. com) have been performed. Considering that, unlike ZEMAX, various aberration effects are not taken into account with thin lens approximation, in this section it is investigated how close the calculations are in reality when derived in comparison with real ray-tracing simulations.

The geometrical properties of the simulated lidar system used as input parameters in paraxial approximation lead to a DFO $=257 \mathrm{~m}$. More precisely, a laser with initial parameters $\mathrm{DL}=8 \mathrm{~mm}$ and $\mathrm{LBD}=0.8 \mathrm{mrad}$ was considered and expanded by an ideal laser beam expansion unit $(E X=\times 4)$. The expansion unit finally results in an emitted laser beam with a diameter of $32 \mathrm{~mm}$ and divergence LBD $=0.2 \mathrm{mrad}$. Moreover, the laser beam is considered to be tilted towards the telescope central axis with an angle of $A_{\text {tilt }}=0.4 \mathrm{mrad}$.
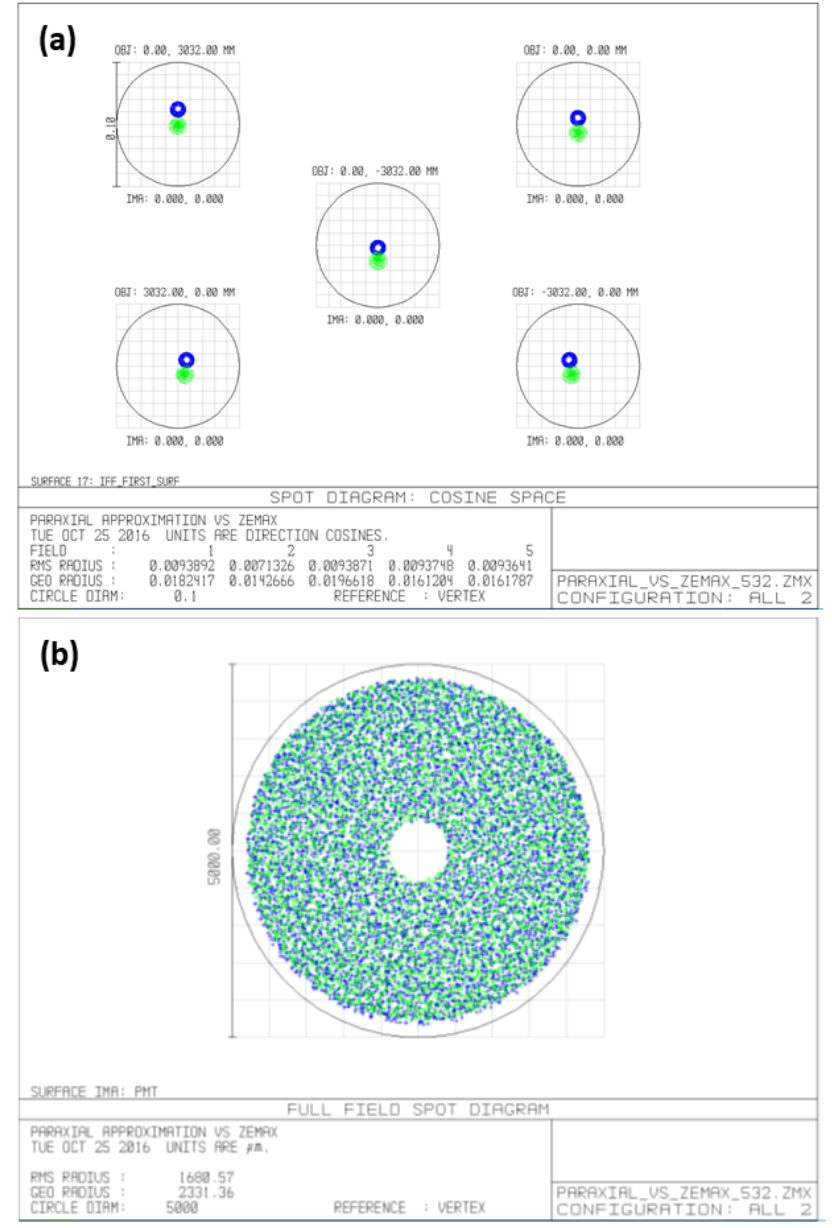

Figure 4. Spot diagrams of far- (green; $10000 \mathrm{~m}$ ) and near- (blue; $257 \mathrm{~m}$ ) range rays on the (a) front surface of the IFF from five different positions within the receiver field of view, and (b) PMT detector with $5 \mathrm{~mm}$ effective diameter (black circle).

The laser light scattered in the atmosphere is collected by an ideal telescope with $\mathrm{DT}=300 \mathrm{~mm}$ and $\mathrm{FT}=600 \mathrm{~mm}$, guided through a circular field stop $\left(D_{\mathrm{FS}}=1.5 \mathrm{~mm}\right)$ to the collimator. The distance $Z_{1}$ has to be as high as possible to mount all the needed optical elements (i.e., beam splitters), always keeping $A_{\max }$ lower than $A_{\mathrm{IFF}}^{\max }$. Considering a receiving system with $\mathrm{RFOV}=1.25 \mathrm{mrad}$, effective $D_{\text {obj. }}=23.5 \mathrm{~mm}$ and a reasonably low $A_{\max }$ value $\left(A_{\max }=1.15^{\circ}\right)$, a distance of $Z_{1}=160 \mathrm{~mm}$, is estimated through Eq. (8). Moreover, for these values Eq. (2) implies that the focal length of the collimator should be $F_{\text {col }}=37.36 \mathrm{~mm}$.

The 3-D ray-tracing simulations have been initiated at $532 \mathrm{~nm}$ using the aforementioned values and assuming an optimized Newtonian telescope. The curvature radii of the primary mirror was set to $1200 \mathrm{~mm}$ and its radius to $150 \mathrm{~mm}$. The surface type was set to a sphere and the conic constant -1 . At $500 \mathrm{~mm}$ above the primary mirror, a secondary diagonal mirror was set with a radius of $25 \mathrm{~mm}$. The 
Table 1. The optical parameters (distances and focal lengths) estimated with paraxial approximation and simulated by ZEMAX, along with their relative differences.

\begin{tabular}{llrrr}
\hline $\begin{array}{l}\text { Optical } \\
\text { parameter }\end{array}$ & $\begin{array}{l}\text { Lens model used from ZEMAX DB } \\
\text { (number of items) }\end{array}$ & ZEMAX & Paraxial & $\begin{array}{r}\text { Relative difference } \\
(\%)\end{array}$ \\
\hline$F_{\text {col }}(\mathrm{mm})$ & SPX019- Newport (x2) & 37.72 & 37.36 & 0.95 \\
$Z_{1}(\mathrm{~mm})$ & & 160 & 160 & 0 \\
$A_{\max }(\mathrm{mrad})$ & & 1.126 & 1.15 & -2.08 \\
$Z_{2}(\mathrm{~mm})$ & & 85.64 & 85.64 & 0 \\
$F_{\text {obj }}(\mathrm{mm})$ & 01LUP033 - Melles Griot (x1) & 74.69 & 70.54 & 5.56 \\
$Z_{3}(\mathrm{~mm})$ & 01LUP031 - Melles Griot (x1) & & & 13 \\
$F_{\text {eye }}(\mathrm{mm})$ & 01LUP009-Melles Griot (x2) & 15.31 & 12.82 & 1.38 \\
\hline
\end{tabular}

backscattering from the laser beam was simulated by a disk of source rays, which was placed at distances of $257 \mathrm{~m}$ and $10000 \mathrm{~m}$ from the telescope. Assuming that the telescope and the laser beam are at the same level (Fig. 1b), then the initial distance between their central axis was considered to be $\mathrm{DTL}=180 \mathrm{~mm}$. However, as the laser beam propagates in the atmosphere with a tilting angle of 0.4 mard, the distance between the laser beam and telescope central axis decreases with a constant rate of $A_{\text {tilt }}$. For each distance, the size of the laser disk was calculated from the LBD. Regarding the optical components mounted after the field stop, we used lenses available from the ZEMAX database, with parameters (i.e., effective focal length and diameter) similar to the ones revealed from the paraxial approximation calculations. More precisely, x2 Newport (model SPX019) lenses were used as collimator (effective $F_{\mathrm{col}}=37.72 \mathrm{~mm}$ and $D_{\text {col }}=25.40 \mathrm{~mm}$ ), x2 Melles Griot (models 01LUP033 and 01LUP031) were used as an objective (effective $F_{\text {obj }}=74.69$ and $D_{\text {obj }}=25.00 \mathrm{~mm}$ ) and x2 Melles Griot (model 01LUP009) lenses were used as an eyepiece lens (effective $F_{\text {eye }}=15.31$ and $D_{\text {eye }}=12.50 \mathrm{~mm}$; Table 1 ). In ZEMAX we set the distance between the collimator and the IFF at exactly $160 \mathrm{~mm}\left(Z_{1}\right)$, the eyepiece at $85.64 \mathrm{~mm}$ after the IFF $\left(Z_{2}\right)$, while the distance between the eyepiece and PMT was at $13 \mathrm{~mm}$. The value of distance $Z_{3}$ was not kept constant in the ZEMAX simulation as it was estimated from the paraxial calculations. Instead, this distance was slightly increased up to some millimeters in order to sufficiently image the telescope aperture on the PMT $\left(D_{\mathrm{PMT}}=5 \mathrm{~mm}\right)$, without any truncation of the rays. Please note here that, in the case of real simulations with ZEMAX, the lenses cease to be ideal thin lenses. The distances, namely $Z_{1}$, $Z_{2}$ and $Z_{3}$, are measured from the points on the principal axis of the last surface of each lens up to the first surface of the next optical component. For example, the measured distance $Z_{1}$ refers to the distance between the last surface of the collimator up to the first surface of the IFF filter, located on the principal axis. In Fig. 4 the spot diagrams of rays from far (green spots; $10000 \mathrm{~m}$ ) and near range (blue spots; $257 \mathrm{~m}$ ) are demonstrated. The five field points selected so as to make an object height with radius equal to LBD $x Z+\frac{\mathrm{DL}}{2}$, where $Z$ is the atmospheric distance from the lidar. The central field point is located at the center of the disk, with coordinates $\left(x_{C}, y_{C}\right)=\left(0, \mathrm{DTL}-Z \times A_{\text {tilt }}\right)$, with respect to the Cartesian system shown in Fig. 1a, while the remaining four are selected to be on the perimeter of this disk with coordinates $\left(x_{R}, y_{R}\right)=$ $\left(-Z \times \mathrm{LBD}, y_{C}\right), \quad\left(x_{\mathrm{L}} y_{L}\right)=\left(Z \times \operatorname{LBD}, y_{C}\right),\left(x_{\mathrm{U}}, y_{U}\right)=$ $\left(0, y_{C}+Z \times \mathrm{LBD}\right),\left(x_{D}, y_{D}\right)=\left(0, y_{C}-Z \times \mathrm{LBD}\right) . \quad$ The spot diagrams in Fig. 4 a are in cosine space, demonstrating the angle with which each field point of far- and near-range rays falls on the first surface of the IFF filter. The maximum field incident angle on the IFF was found to be equal to $0.0196 \mathrm{mrad}$. The full field spot diagram demonstrated in Fig. 4b refers to the surface of the PMT. As can be seen in Fig. $4 \mathrm{~b}$ a homogeneous distribution of far- and near-range rays on PMT surface have been achieved, covering the same area. The spot diameter was found to be $4.6 \mathrm{~mm}$, within the $5 \mathrm{~mm}$ diameter of effective detector aperture, revealing an overall sufficient imaging of far- and near-range rays on the detector.

The relative differences between the calculated parameters from paraxial approximation and the simulations with ZEMAX are demonstrated in Table 1, and the slight discrepancies are attributed to the following reasons: (a) the slightly different parameters of lenses used from ZEMAX database compared to those used as input $\left(F_{\mathrm{col}}\right)$ or estimated $\left(F_{\text {obj }} F_{\text {eye }}\right)$ with the paraxial approximation formulation and (b) the inability of the paraxial approximation to take into account the sag of each lens surface to better model the refraction of off-axis rays in contrast to the ZEMAX simulation. However, the effect of the telescope's defocus seems to be taken into account by paraxial optics. Rays arriving at the telescope from near points intersect behind the focal plane. Where they will finally intersect depends on how near the origin point is. Here the near points are considered to be from $257 \mathrm{~m}$ (blue lines), which they intersect at $1.4 \mathrm{~mm}$ behind the focal plane of the telescope. As the points are taken farther away, the intersection point will tend to approach the focal plane, eventually being virtually on the focal plane for 


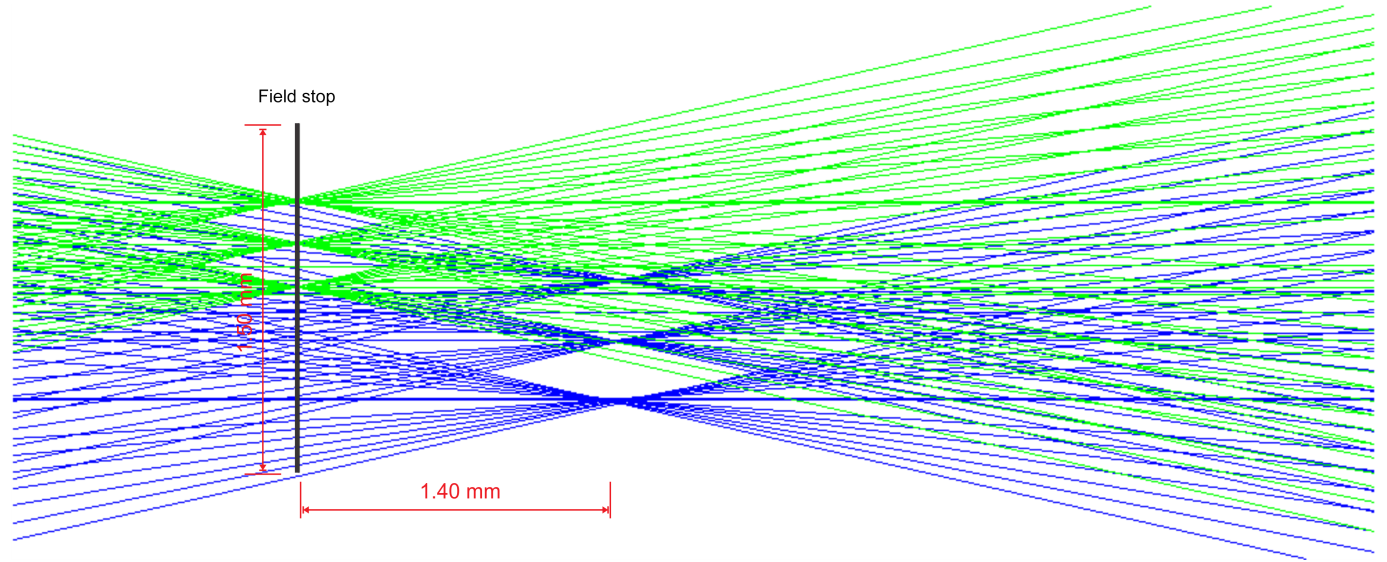

Figure 5. ZEMAX simulations regarding the focal plane of the telescope where the field stop $\left(D_{\mathrm{FS}}=15 \mathrm{~mm}\right)$ is placed. The focal plane is where parallel rays will intersect after passing through the telescope. Rays arriving at the telescope from near points will intersect behind the focal plane. Where they finally intersect depends on how near the origin point is. Rays originating from points at $257 \mathrm{~m}$ from the lidar (blue lines) intersect at $1.4 \mathrm{~mm}$ behind the focal plane of the telescope. As the points are taken farther away, the intersection point will tend to approach the focal plane, eventually being virtually on the focal plane for long enough distances (i.e., the points from $10000 \mathrm{~m}$ considered here with green lines).

long enough distances (i.e., the points from $10000 \mathrm{~m}$ considered here with green lines). From the paraxial formula relating object and image positions, a simple calculation shows that, for a thin lens of $600 \mathrm{~mm}$ focal length, the image of a point at $10000 \mathrm{~m}$ from the lens will be at $600.04 \mathrm{~mm}$ from the lens plane, while the image of a point at $257 \mathrm{~m}$ will be at $601.40 \mathrm{~mm}$, the difference being $1.36 \mathrm{~mm}$, very close to the $1.40 \mathrm{~mm}$ revealed by ZEMAX simulations (Fig. 5). Thus, the telescope processes the rays according to the paraxial optics laws (or at least to a good degree of approximation), so the considered rays satisfy the paraxial conditions.

\section{Summary and conclusions}

Based on thin lens approximation formulas, a set of equations is derived, describing the optical design of a typical EARLINET lidar system. The limitations of a lidar optical setup are revealed through geometric optics, from the emitted laser beam to the projection of the entrance pupil on the photomultiplier. The main lidar issue studied here concerns the distance of full overlap and how this depends on the entire geometry which describes the optical path in the detection unit of a lidar system, not only on the laser telescope geometry. The usage of IFF with small bandwidth for background suppression is limited by their small acceptance angle, especially if the alignment uncertainties of the mechanical setup of the lidar optics are taken into account. The evaluation of the paraxial approximation formulation has been performed with ZEMAX ray-tracing simulations, showing an overall good performance with a relative difference (between ZEMAX and paraxial approximation) of the order of 5\% (see Table 1) and a negligible impact on the system performance.
These differences are mainly attributed to the inability of thin lens formulations to better model the refraction of the off-axis rays. The described formulation cannot substitute an advanced optical design software, since 3-D ray-tracing simulations of realistic lidar systems are required to reveal the necessity of using the highest-quality optical parts mounted with the highest possible accuracy.

Data availability. The Excel worksheet that integrates the entire set of equations as described in this paper is available upon request from the author (panko@noa.gr). 


\section{Appendix A}

The center wavelength $\lambda_{o}$ of an interference filter (IFF) is shifted to $\lambda_{\mathrm{s}}$ with an incident angle $A_{\mathrm{IFF}}$ according to

$\frac{\lambda_{\mathrm{s}}}{\lambda_{o}}=\sqrt{1-\left[\frac{n_{o}}{n_{\mathrm{e}}} \times \sin \left(A_{\mathrm{IFF}}\right)\right]^{2}}$,

with the effective refractive index of the filter $n_{\mathrm{e}}$ and the refractive index of the environment $n_{o}$. The shift is to smaller wavelengths with increasing $A_{\mathrm{IFF}}$, and larger $n_{\mathrm{e}}$. Examples for IFF are a Barr filter with $0.5 \mathrm{~nm}$ bandwidth (BWFWHM) at $532 \mathrm{~nm}, n_{\mathrm{e}}=1.99$ and a temperature coefficient of $0.0021 \mathrm{~nm}^{\circ} \mathrm{C}^{-1}$, as well as an Andover filter with $\mathrm{BW}=0.15 \mathrm{~nm}$ at $532 \mathrm{~nm}, n_{\mathrm{e}}=1.45$ and temperature coefficient $0.016 \mathrm{~nm}^{\circ} \mathrm{C}^{-1}$. The incident angles $A_{\mathrm{IFF}}$ are limited by the maximum allowed wavelength shift for acceptable transmission, which have been set to $0.7 \times \frac{\mathrm{BW}}{2}$, i.e., about $0.18 \mathrm{~nm}$ (Barr) and $0.05 \mathrm{~nm}$ (Andover). This results in $A_{\mathrm{IFF}}^{\max }$ of 2.9 and $1.14^{\circ}$ for Barr and Andover filters respectively.

\section{Appendix B: A list of the abbreviations that are used for describing the lidar parameters, along with their meaning}

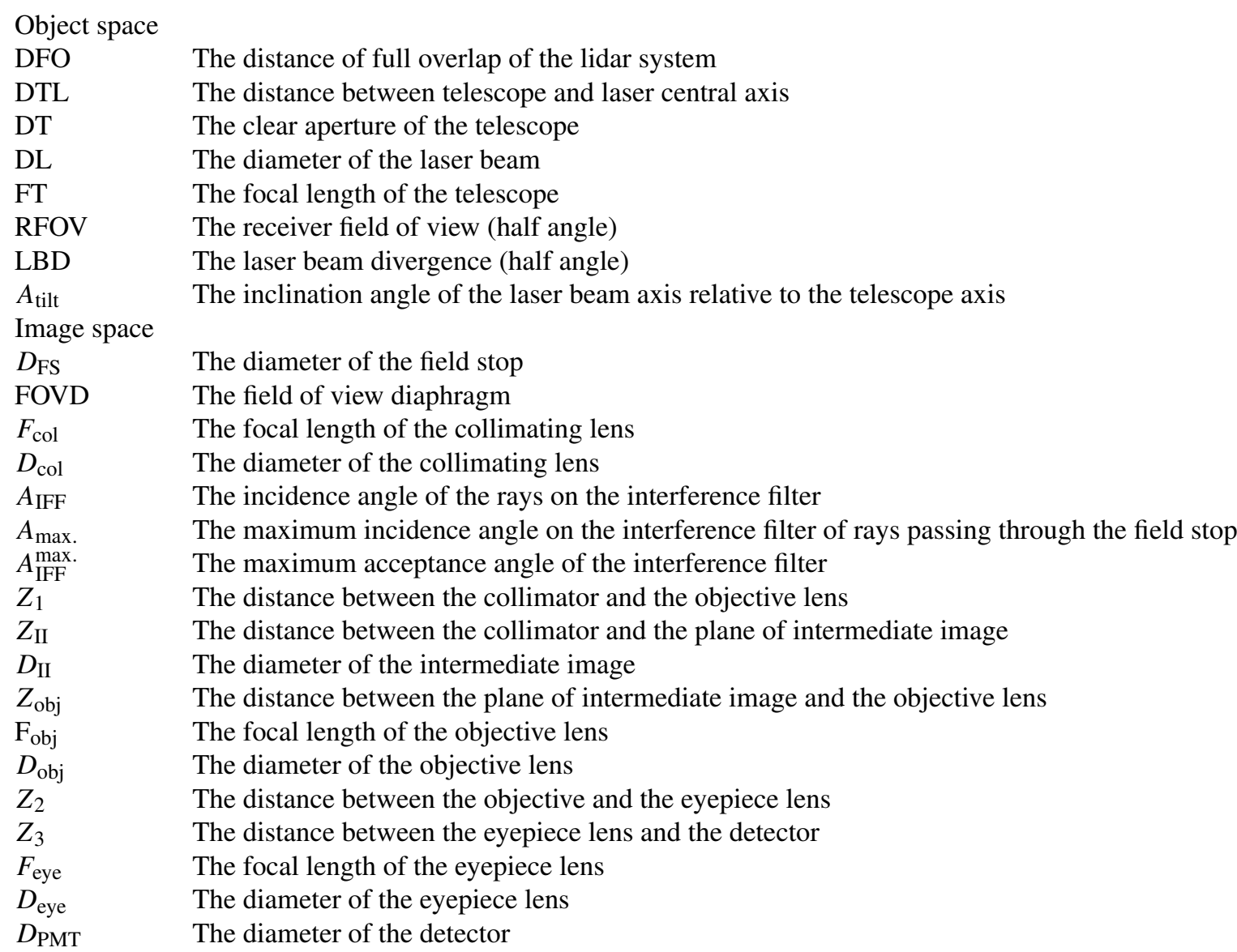


Competing interests. The authors declare that they have no conflict of interest.

Special issue statement. This article is part of the special issue "EARLINET, the European Aerosol Research Lidar Network". It is not associated with a conference.

Acknowledgements. I would like to thank Volker Freudenthaler for the helpful discussions, ideas and useful comments.

The publication was supported by the European Union Seventh Framework Programme (FP7-REGPOT-2012-2013-1) in the framework of the project BEYOND, under grant agreement no. 316210 (BEYOND-Building Capacity for a Center of Excellence for EO-based monitoring of Natural Disasters).

Edited by: Albert Ansmann

Reviewed by: two anonymous referees

\section{References}

Abramochkin, A. I. and Tikhomirov, A. A.: Optimization of a lidar receiving system 2, Spatial filters, Atmos. Ocean. Opt. 12, 331342, 1999.

Amiridis, V., Melas, D., Balis, D. S., Papayannis, A., Founda, D., Katragkou, E., Giannakaki, E., Mamouri, R. E., Gerasopoulos, E., and Zerefos, C.: Aerosol Lidar observations and model calculations of the Planetary Boundary Layer evolution over Greece, during the March 2006 Total Solar Eclipse, Atmos. Chem. Phys., 7, 6181-6189, https://doi.org/10.5194/acp-7-6181-2007, 2007.

Ansmann, A., Riebesell, M., and Weitkamp, C.: Measurement of atmospheric aerosol extinction profiles with a Raman lidar, Opt. Lett., 15, 746-748, https://doi.org/10.1364/OL.15.000746, 1990.

Ansmann, A., Wandinger, U., Riebesell, M., Weitkamp, C., and Michaelis, W.: Independent measurement of extinction and backscatter profiles in cirrus clouds by using a combined Raman elastic-backscatter lidar, Appl. Opt., 31, 7113-7131, https://doi.org/10.1364/AO.31.007113, 1992.

Baars, H., Ansmann, A., Engelmann, R., and Althausen, D.: Continuous monitoring of the boundary-layer top with lidar, Atmos. Chem. Phys., 8, 7281-7296, https://doi.org/10.5194/acp-8-72812008, 2008.

Belegante, L., Bravo-Aranda, J. A., Freudenthaler, V., Nicolae, D., Nemuc, A., Alados-Arboledas, L., Amodeo, A., Pappalardo, G., D’Amico, G., Engelmann, R., Baars, H., Wandinger, U., Papayannis, A., Kokkalis, P., and Pereira, S. N.: Experimental assessment of the lidar polarizing sensitivity, Atmos. Meas. Tech. Discuss., https://doi.org/10.5194/amt-2015-337, in review, 2016.

Bösenberg, J.: Study on retrieval algorithms for a backscatter lidar, Final report, Max-Plank- Inst. Für Meteorol., Hamburg, Germany, MPI-Rep. 226, 1997.

Bravo-Aranda, J. A., Belegante, L., Freudenthaler, V., AladosArboledas, L., Nicolae, D., Granados-Muñoz, M. J., GuerreroRascado, J. L., Amodeo, A., D’Amico, G., Engelmann, R., Pappalardo, G., Kokkalis, P., Mamouri, R., Papayannis, A., NavasGuzmán, F., Olmo, F. J., Wandinger, U., Amato, F., and Haeffelin, M.: Assessment of lidar depolarization uncertainty by means of a polarimetric lidar simulator, Atmos. Meas. Tech., 9, 49354953, https://doi.org/10.5194/amt-9-4935-2016, 2016.

Chourdakis, G., Papayannis, A., and Porteneuve, J.: Analysis of the receiver response for a noncoaxial lidar system with fiber-optic output, Appl. Opt., 41, 2715-2723, 2002.

Comeron, A., Sicard, M., Kumar, D., and Rocadenbosch, F.: Use of a field lens for improving the overlap function of a lidar system employing an optical fiber in the receiver assembly, Appl. Opt., 50, 5538-5544, 2011.

Dho, S. W., Park, Y. J., and Kong, H. J.: Experimental determination of a geometric form factor in a lidar equation for an inhomogeneous atmosphere, Appl. Opt., 36, 6009-6010, https://doi.org/10.1364/AO.36.006009, 1997.

Eloranta, E. W.: Practical model for the calculation of multiply scattered lidar returns, Appl. Opt., 37, 2464-2472, https://doi.org/10.1364/AO.37.002464, 1998.

Engelmann, R., Kanitz, T., Baars, H., Heese, B., Althausen, D., Skupin, A., Wandinger, U., Komppula, M., Stachlewska, I. S., Amiridis, V., Marinou, E., Mattis, I., Linné, H., and Ansmann, A.: The automated multiwavelength Raman polarization and water-vapor lidar Polly XT: the neXT generation, Atmos. Meas. Tech., 9, 1767-1784, https://doi.org/10.5194/amt-9-1767-2016, 2016.

Fernald, F. G., Herman, B. M., and Reagan, J. A.: Determination of Aerosol Height Distribution by Lidar, J. Appl. Meteorol., 11, 482-489, 1972.

Freudenthaler, V: Optimized background suppression in near field lidar telescopes, in: Proceedings 5 of the 6th International Symposium on Tropospheric Profiling: Needs and Technologies (ISTP 2003), edited by: Wandinger, U., Engelmann, R., and Schmieder, K., Institute for Tropospheric Research, 243-245, 2003.

Freudenthaler, V.: Effects of spatially inhomogeneous photomultiplier sensitivity on LIDAR signals and remedies, in: Proceedings of the 22nd International Laser Radar Conference (ILRC 2004) held 12-16 July 2004 in Matera, Italy, edited by: Pappalardo, G. and Amodeo, A., European Space Agency, Paris, 37 pp., ESA SP-561, 2004.

Freudenthaler, V.: The telecover test: a quality assurance tool for the optical part of a lidar system, in: Reviewed and Revised Papers Presented at the 24th International Laser Radar Conference, Boulder, Colorado, USA, 23-27 June 2008, Boulder, Colorado, USA, presentation: S01P-30, available at: http://epub.ub. uni-muenchen.de/12958/ (last access: 8 July 2016), 2008.

Freudenthaler, V.: About the effects of polarising optics on lidar signals and the $\Delta 90$ calibration, Atmos. Meas. Tech., 9, 41814255, https://doi.org/10.5194/amt-9-4181-2016, 2016.

Freudenthaler, V., Esselborn, M., Wiegner, M., Heese, B., Tesche, M., Ansmann, A., Müller, D., Althausen, D., Wirth, M., Fix, A., Ehret, G., Knippertz, P., Toledano, C., Gasteiger, J., Garhammer, M., and Seefeldner, M.: Depolarization ratio profiling at several wavelengths in pure Saharan dust during SAMUM 2006, Tellus B, 61, 165-179, https://doi.org/10.1111/j.16000889.2008.00396.x, 2009.

Guerrero-Rascado, J. L., Costa, M. J., Bortoli, D., Silva, A. M., Lyamani, H., and Alados-Arboledas, L.: Infrared lidar overlap function: an experimental determination, Opt. Express, 18, 2035020359, 2010. 
Haarig, M., Engelmann, R., Ansmann, A., Veselovskii, I., Whiteman, D. N., and Althausen, D.: $1064 \mathrm{~nm}$ rotational Raman lidar for particle extinction and lidar-ratio profiling: cirrus case study, Atmos. Meas. Tech., 9, 4269-4278, https://doi.org/10.5194/amt9-4269-2016, 2016.

Halldórsson, T. and Langerholc, J.: Geometrical form factors for the lidar function, Appl. Opt., 17, 240-244, https://doi.org/10.1364/AO.17.000240, 1978.

Hamamatsu Photonics: Photomultiplier tubes Basics and Applications, 2 Edn., 1-305, 2006.

Iarlori, M., Madonna, F., Rizi, V., Trickl, T., and Amodeo, A.: Effective resolution concepts for lidar observations, Atmos. Meas. Tech., 8, 5157-5176, https://doi.org/10.5194/amt-8-5157-2015, 2015.

Jenness, J. R., Lysak, D. B., and Philbrick, C. R.: Design of a lidar receiver with fiber-optic output, Appl. Opt., 36, 4278-4284, 1997.

Klett, J. D.: Stable analytical inversion solution for processing lidar returns, Appl. Opt., 20, 211-220, https://doi.org/10.1364/AO.20.000211, 1981.

Matthias, V. and Bösenberg, J.: Aerosol climatology for the planetary boundary layer derived from regular lidar measurements, Atmos. Res., 63, 221-245, 2002.

Matthias, V., Balis, D., Bösenberg, J., Eixmann, R., Iarlori, M., Komguem, L., Mattis, I., Papayannis, A., Pappalardo, G., Perrone, M. R., and Wang, X.: Vertical aerosol distribution over Europe: Statistical analysis of Raman lidar data from 10 European Aerosol Research Lidar Network (EARLINET) stations, J. Geophys. Res., 109, D18201, https://doi.org/10.1029/2004JD004638, 2004.

Mattis, I., Tesche, M., Grein, M., Freudenthaler, V., and Müller, D.: Systematic error of lidar profiles caused by a polarization-dependent receiver transmission: quantification and error correction scheme, Appl. Opt., 48, 2742-2751, https://doi.org/10.1364/AO.48.002742, 2009.

Müller, D., Wandinger, U., and Ansmann, A.: Microphysical particle parameters from extinction and backscatter lidar data by inversion with regularization: theory, Appl. Opt., 38, 2346-2357, https://doi.org/10.1364/AO.38.002346, 1999.

Pappalardo, G., Amodeo, A., Apituley, A., Comeron, A., Freudenthaler, V., Linné, H., Ansmann, A., Bösenberg, J., D’Amico, G., Mattis, I., Mona, L., Wandinger, U., Amiridis, V., AladosArboledas, L., Nicolae, D., and Wiegner, M.: EARLINET: towards an advanced sustainable European aerosol lidar network, Atmos. Meas. Tech., 7, 2389-2409, https://doi.org/10.5194/amt7-2389-2014, 2014.

Sasano, Y., Shimizu, H., Takeuchi, N., and Okuda, M.: Geometrical form factor in the laser radar equation: an experimental determination, Appl. Opt., 18, 3908-3910, https://doi.org/10.1364/AO.18.003908, 1979.
Sassen, K.: Polarization in Lidar, in Lidar, edited by: Weitkamp, D. C., 19-42, Springer New York, available at: http://link.springer. com/chapter/10.1007/0-387-25101-4_2 (last access: 29 March 2016), 2005.

Simeonov, V., Larcheveque, G., Quaglia, P., van den Bergh, H., and Calpini, B.: Influence of the photomultiplier tube spatial uniformity on lidar signals, Appl. Opt., 38, 5186-5190, https://doi.org/10.1364/AO.38.005186, 1999.

Stelmaszczyk, K., Dell'Aglio, M., Chudzyński, S., Stacewicz, T., and Wöste, L.: Analytical function for lidar geometrical compression form-factor calculations, Appl. Opt., 44, 1323-1331, 2005.

Tomine, K., Hirayama, C., Michimoto, K., and Takeuchi, N.: Experimental determination of the crossover function in the laser radar equation for days with a light mist, Appl. Opt., 28, 2194-2195, https://doi.org/10.1364/AO.28.002194, 1989.

Veselovskii, I., Kolgotin, A., Griaznov, V., Müller, D., Wandinger, U., and Whiteman, D. N.: Inversion with regularization for the retrieval of tropospheric aerosol parameters from multiwavelength lidar sounding, Appl. Opt., 41, 3685-3699, https://doi.org/10.1364/AO.41.003685, 2002.

Veselovskii, I., Dubovik, O., Kolgotin, A., Lapyonok, T., Di Girolamo, P., Summa, D., Whiteman, D. N., Mishchenko, M., and Tanré, D.: Application of randomly oriented spheroids for retrieval of dust particle parameters from multiwavelength lidar measurements, J. Geophys. Res.-Atmos., 115, D21203, https://doi.org/10.1029/2010JD014139, 2010.

Veselovskii, I., Whiteman, D. N., Korenskiy, M., Suvorina, A., and Pérez-Ramírez, D.: Use of rotational Raman measurements in multiwavelength aerosol lidar for evaluation of particle backscattering and extinction, Atmos. Meas. Tech., 8, 41114122, https://doi.org/10.5194/amt-8-4111-2015, 2015.

Wandinger, U.: Multiple-scattering influence on extinction-and backscatter-coefficient measurements with Raman and highspectral-resolution lidars, Appl. Opt., 37, 417-427, 1998.

Wandinger, U.: Introduction to Lidar, in Lidar, edited by: Weitkamp, D. C., 1-18, Springer New York, available at: http://link. springer.com/chapter/10.1007/0-387-25101-4_1 (last access: 8 July 2016), 2005.

Wandinger, U. and Ansmann, A.: Experimental determination of the lidar overlap profile with Raman lidar, Appl. Opt., 41, 511-514, https://doi.org/10.1364/AO.41.000511, 2002.

Whiteman, D. N.: Application of statistical methods to the determination of slope in lidar data, Appl. Opt., 38, 3360-3369, https://doi.org/10.1364/AO.38.003360, 1999. 